\title{
ANL/ASD/CP-102684
}

\section{Quasilinear theory of high-gain FEL saturation}

\author{
N. A. Vinokurov ${ }^{\text {la }}$, Z. Huang ${ }^{b}$, O. A. Shevchenko ${ }^{a}$, and K.-J. Kim ${ }^{b}$ \\ ${ }^{a}$ Budker Institute of Nuclear Physics, 11 Ac. Lavrentyev Prosp., 630090, Novosibirsk, Russia \\ ${ }^{b}$ Advanced Photon Source, Argonne National Laboratory, 9700 S. Cass Avenue, \\ Argonne, IL 60439, USA
}

\begin{abstract}
Understanding of saturation behavior is important to assess the performance of a highgain free-electron laser (FEL). In this paper we study the saturation mechanism using a quasilinear approximation to the coupled Maxwell-Vlasov equations. It is found that the quasilinear theory correctly describes the evolution of the radiation field from the small signal regime to reaching saturation.
\end{abstract}

Keywords: Free-electron laser

\section{Introduction}

The electron beam traveling through the long undulator is unstable with respect to the longitudinal bunching at the frequency of the spontaneous undulator radiation in the forward direction. For small bunching the system is linear, and the bunching grows exponentially along the undulator. But when the value of bunching becomes not so small, the exponential growth stops. This phenomenon is referred to as "saturation." The reason for saturation is the obvious fact that the value of bunching cannot exceed 1 , as the distribution function of particles is positive. In other words, the amplitude of the AC component of the electron current cannot exceed its DC component more than twice. From the point of view of the linear small-signal theory, the growth of a particular harmonic of the distribution function is limited by the nonlinear interaction with other harmonics. Sometimes the interaction with the zero harmonics (the average) dominates. The beam energy spread increases and therefore the bunching growth rate decreases. This

\footnotetext{
${ }^{1}$ Corresponding author. email: vinokurov@inp.nsk.su
}

The submitted manuscript has been created by the University of Chicago as Operator of Argonne National Laboratory ("Argonne") under Contract No. W-31-109-ENG-38 with the U.S. Department of Energy. The U.S. Government retains for itself, and others acting on its behalf, a paid-up, nonexclusive. irrevocable worldwide license in said article to reproduce, prepare derivative works, distribute copies to the public, and perform publicly and display publicly, by or on behalf of the Government. 


\section{DISCLAIMER}

This report was prepared as an account of work sponsored by an agency of the United States Government. Neither the United States Government nor any agency thereof, nor any of their employees, make any warranty, express or implied, or assumes any legal liability or responsibility for the accuracy, completeness, or usefulness of any information, apparatus, product, or process disclosed, or represents that its use would not infringe privately owned rights. Reference herein to any specific commercial product, process, or service by trade name, trademark, manufacturer, or otherwise does not necessarily constitute or imply its endorsement, recommendation, or favoring by the United States Government or any agency thereof. The views and opinions of authors expressed herein do not necessarily state or reflect those of the United States Government or any agency thereof. 


\section{DISCLAIMER}

Portions of this document may be illegible in electronic image products. Images are produced from the best available original document. 
mechanism is well known in plasma physics as the quasilinear relaxation (see for example [1]).

In this paper we derive the quasilinear equations for 1-D free-electron laser (FEL) theory and solve them numerically. It is shown that for large enough initial energy spread the maximum bunching is much less than one, and so the quasilinear approximation is applicable until the saturation length.

\section{Derivation of quasilinear equations}

The FEL equations may be obtained from the Maxwell equations and continuity equation in the phase space (Vlasov equation). To simplify the consideration we assume the electromagnetic wave to be quasimonochromatic, i.e., having a narrow spectrum near frequency $\omega$. It is exactly true if we neglect radiation at higher harmonics of the fundamental frequency (for weak undulator field, for example). Using the standard approach [2] one can easily write down the following equations for the distribution function $f(\psi, \eta, \xi)$ and the dimensionless complex field amplitude $A(\psi, \xi)$ :

$$
\begin{aligned}
& \frac{\partial f}{\partial \xi}+\eta \frac{\partial f}{\partial \psi}-2 \operatorname{Re}\left(A e^{i \psi}\right) \frac{\partial f}{\partial \eta}=0, \\
& \frac{d A}{d \xi}=\frac{1}{2 \pi} \int_{0}^{2 \pi} \int_{-\infty}^{\infty} e^{-i \psi} f d \eta d \psi .
\end{aligned}
$$

Tis

OCT 062990

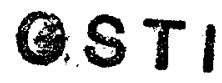

Here $\xi=2 \rho k_{w} z$ is the dimensionless longitudinal coordinate, $k_{w}$ is the undulator wavenumber, $\rho$ is the Pierce parameter, $\eta=\delta \gamma /(\gamma \rho)$ is the relative deviation of particle energy from the synchronous one $\gamma=\left(1+K^{2}\right) \gamma_{\|}=\left(1+K^{2}\right) \sqrt{\frac{\omega}{2 c k_{w}}}, K$ is the undulator deflection parameter, and $\psi=\omega\left[\frac{z}{c}\left(1+\frac{1}{2 \gamma_{\|}^{2}}\right)-t\right]$ is the slow time variable.

In this work we intend to show the applicability of the quasilinear approximation near the saturation point. For further simplification we restrict our consideration to the case of a monochromatic electromagnetic field. It is true for the case of amplification of the monochromatic seed signal at frequency $\omega$. Then the field amplitude $A$ does not depend on time $\psi$, and the distribution function $f$ is assumed to be periodic in $\psi$. 
$f(\psi, \eta, \xi)=\sum_{n=-\infty}^{\infty} f_{n}(\eta, \xi) e^{i n \psi}$ and normalized as $\int_{-\infty}^{\infty} f_{0}(\eta, \xi) d \eta=1$. This assumption allowed us to write down the chain of equations for the harmonics of the distribution function:

$$
\frac{\partial f_{n}}{\partial \xi}+i \eta n f_{n}=A \frac{\partial}{\partial \eta} f_{n-1}+A^{*} \frac{\partial}{\partial \eta} f_{n+1} .
$$

Truncation of Eq.(2) for $n \geq 2$ gives the closed system of equations that may be solved numerically:

$$
\begin{aligned}
& \frac{\partial f_{1}}{\partial \xi}+i \eta f_{1}=A \frac{\partial f_{0}}{\partial \eta} \\
& \frac{\partial f_{0}}{\partial \xi}=2 \operatorname{Re}\left(A^{*} \frac{\partial}{\partial \eta} f_{1}\right), \\
& \frac{d A}{d \xi}=\int f_{1} d \eta
\end{aligned}
$$

\section{Numerical solution}

The numerical solution is based on the usage of the explicit centered difference scheme. Some results for centered Gaussian initial distribution with standard deviation 1 are presented in Figs. 1 and 2. Figure 1 shows the dependence of the field amplitude on the longitudinal coordinate. Note that the efficiency is $-\langle\eta\rangle=|A|^{2}$. One can see that at some value of $\xi$ the exponential growth stops and the field amplitude reaches its first maximum. The cause of this becomes clear after considering the behavior of the average distribution function at different $\xi$ (Fig. 2). The increase of the first harmonic in our approximation leads to the growth of the energy spread, which causes the "saturation." Figure 3 shows the square of bunching $\left|\int f_{1} d \eta\right|^{2}$. It may be seen that its value remains significantly less than one until the first maximum of the field amplitude. This fact confirms the statement that the quasilinear mechanism of saturation dominates at the first stage. 


\section{Values of higher harmonics}

To verify the self-consistency of the proposed quasilinear approximation we need to estimate the values of higher current harmonics. The approximate solution for the second harmonic of the distribution function may be found from Eq. (2):

$$
f_{2}=\int_{0}^{\xi} e^{-2 i \eta\left(\xi-\xi^{\prime}\right)} A\left(\xi^{\prime}\right) \frac{\partial}{\partial \eta} f_{1}\left(\xi^{\prime}, \eta\right) d \xi^{\prime}
$$

Then the "improved" quasilinear system can be written as

$$
\begin{aligned}
& \frac{\partial f_{1}}{\partial \xi}+i \eta f_{1}=A \frac{\partial f_{0}}{\partial \eta}+A^{*} \frac{\partial}{\partial \eta} \int_{0}^{\xi} e^{-2 i \eta\left(\xi-\xi^{\prime}\right)} A\left(\xi^{\prime}\right) \frac{\partial}{\partial \eta} f_{1}\left(\eta, \xi^{\prime}\right) d \xi^{\prime}, \\
& \frac{\partial f_{0}}{\partial \xi}=2 \operatorname{Re}\left(A^{*} \frac{\partial}{\partial \eta} f_{1}\right), \\
& \frac{d A}{d \xi}=\int f_{1} d \eta .
\end{aligned}
$$

The first term on the right side of the first equation in Eq.(3') is proportional to $A$, but the second term is proportional to $A^{3}$. Therefore the contribution of the last term to the value of $f_{1}$ is small at least at the first stage of saturation (before the first field maximum). The second harmonic bunching is plotted in Fig. 3.

\section{Conclusion}

The quasilinear equations describe the saturation phenomena consistently. The calculated normalized efficiencies $-\langle\eta\rangle$ for different values of normalized relative energy spread $\sigma=\sqrt{\left\langle\eta^{2}\right\rangle}$ are shown in Fig. 4. Further investigation on the applicability region of these equations and its comparisons with other models (for example, [3]) is planned.

This work is partly supported by the U. S. Department of Energy, Office of Basic Energy Sciences, under Contract No. W-31-109-ENG-38. 


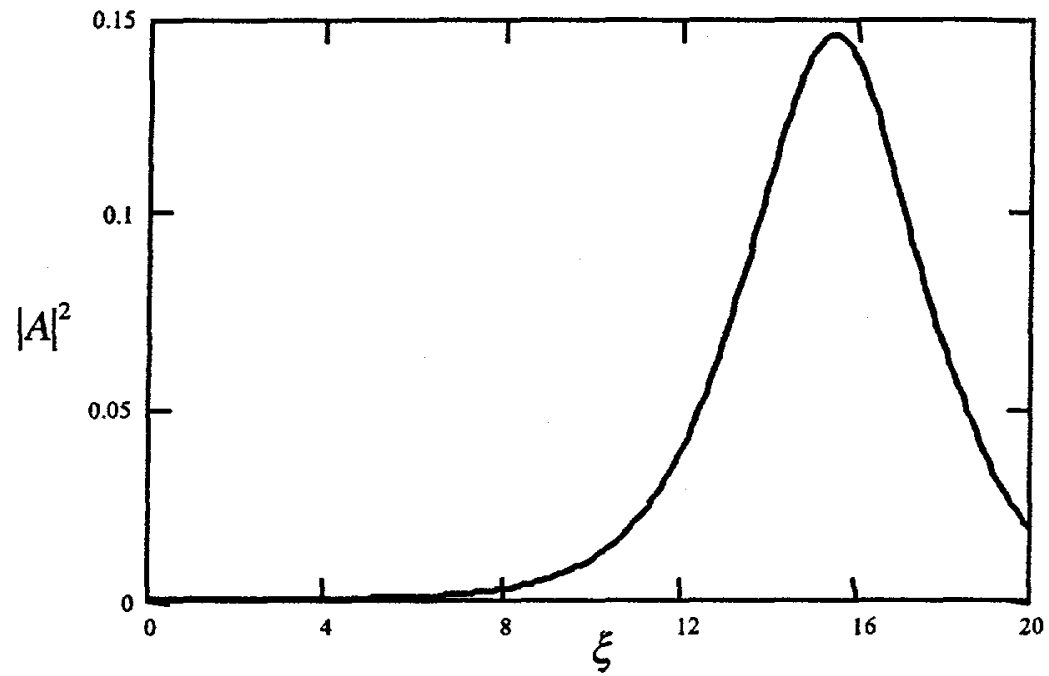

Figure 1. Dependence of the normalized efficiency $|A|^{2}$ on the longitudinal coordinate $\xi$. 

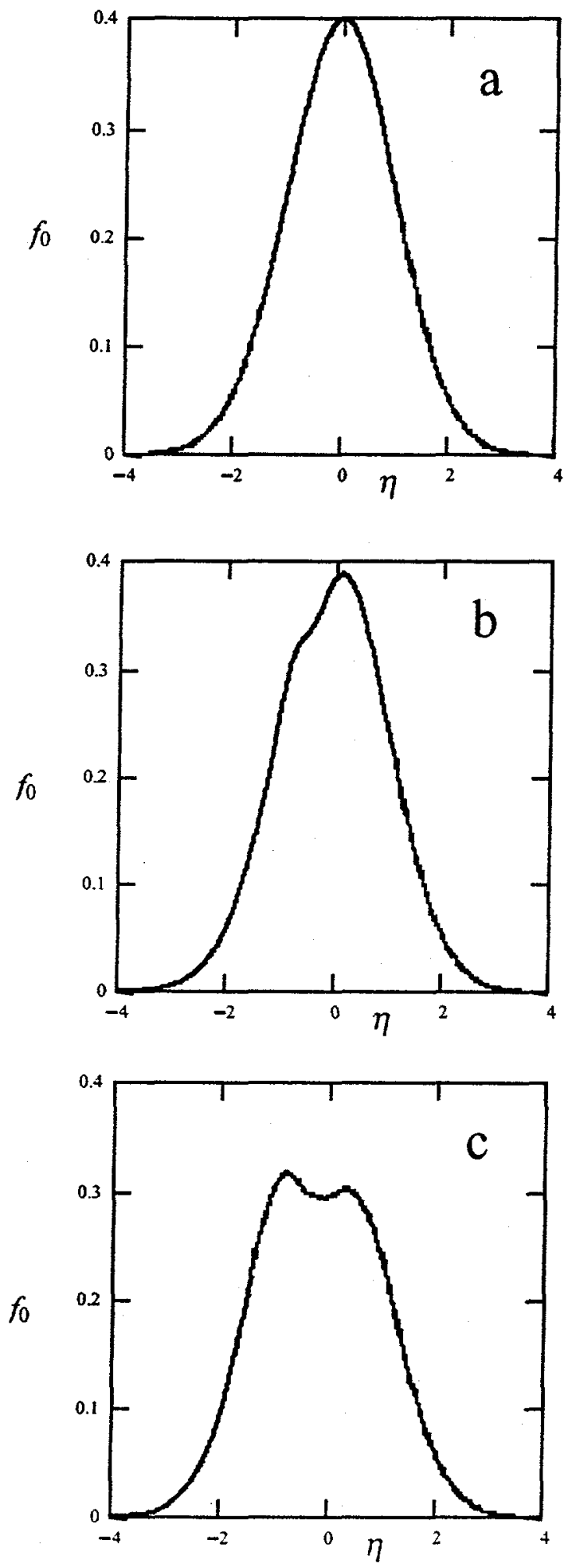

Figure 2. Modification of the distribution function at different stages of the "saturation" process : (a) initial distribution ( $\xi=0)$ : (b) distribution at half of the distance before the 
first maximum ( $\xi=10$ ); (c) distribution at the first maximum of the field amplitude ( $\xi=$ 15.3). 


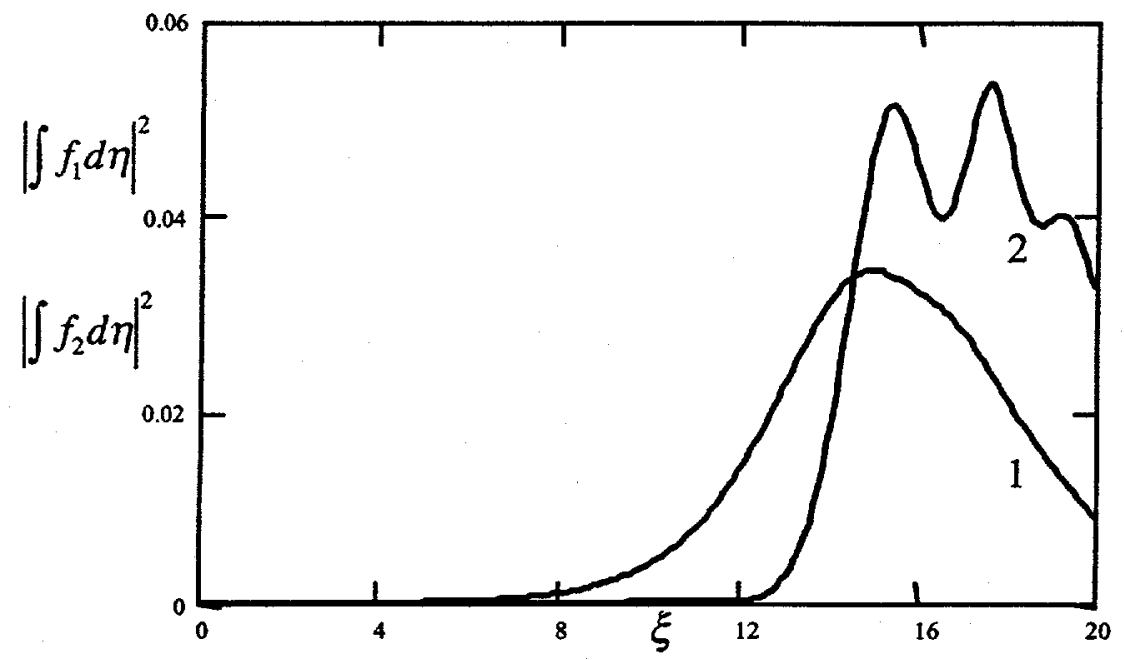

Figure 3. (1) square module of bunching $\left|\int f_{1} d \eta\right|^{2}$ and (2) second harmonic $\left|\int f_{2} d \eta\right|^{2}$ versus distance. 


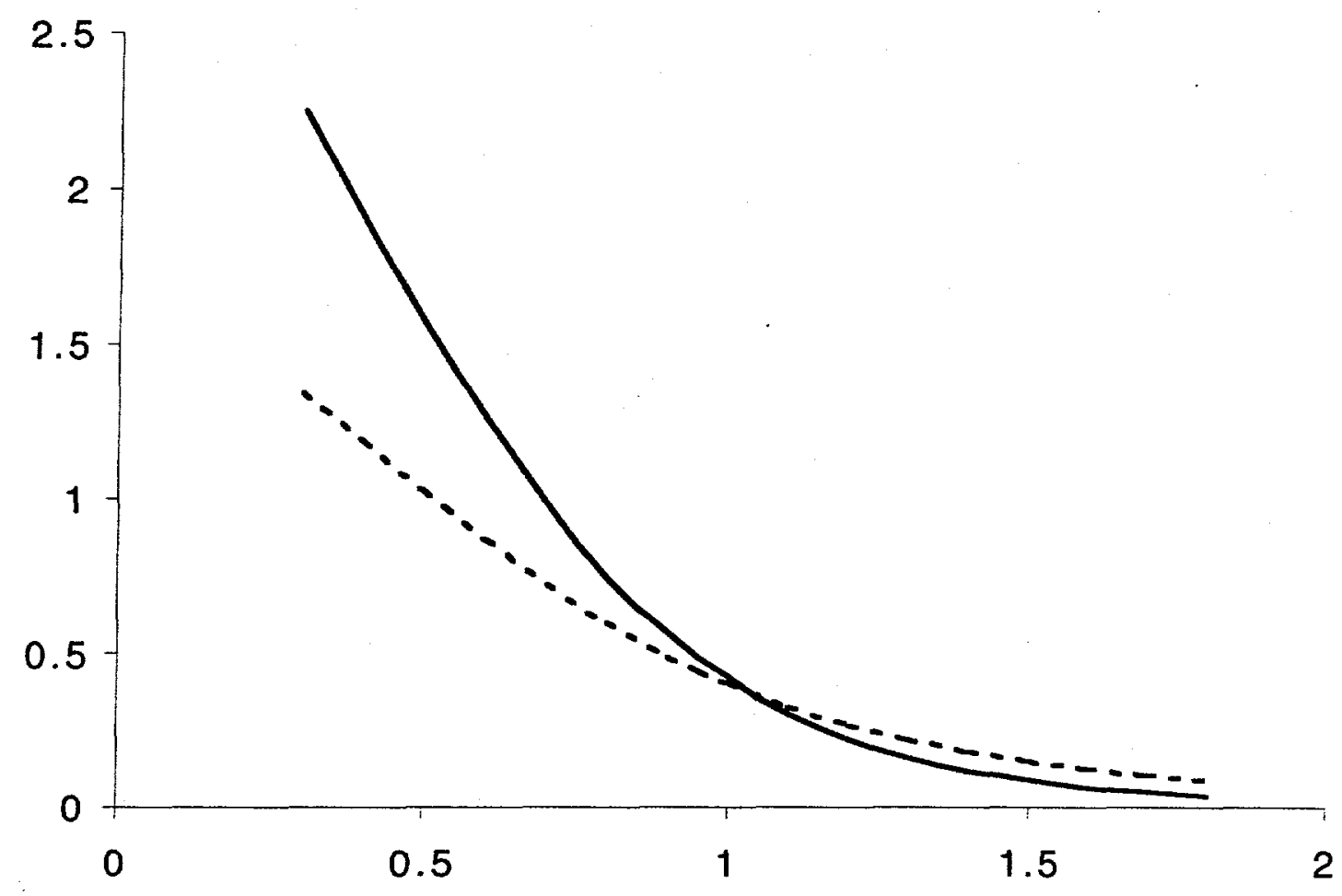

Figure 4. Dependence of the maximum normalized efficiency $\left(|A|^{2}\right)_{\max }$ on the normalized relative energy spread (solid line). Dashed line was obtained using fitting formula [4]. 


\section{References}

1. N. A. Krall, A. W. Trivelpiece, "Principles of Plasma Physics", McGraw-Hill, 1973.

2. K.-J. Kim, Phys Rev. Lett. 57, 1871 (1986).

3. R.L. Gluckstern, S. Krinsky, H. Okamoto, Physical Review E, Vol. 47, N. 6, 4412 (1993).

4. M. Xie, IEEE Proceedings of the 1995 Particle Accelerator Conference, 183 (IEEE, 1996). 